\title{
DERMOELECTROPORATION IN THE TREATMENT OF OVERUSE INJURIES
}

\author{
S. Radev* \\ Department of Physiology, Pathophysiology and Pharmacology, Medical Faculty, Trakia University, \\ Stara Zagora, Bulgaria
}

\begin{abstract}
The musculoskeletal system can suffer from various types of overuse injuries which may affect the bone, muscles, tendons and ligaments. Iliotibial band syndrome (ITBS) is a common overuse injury typically seen in runners, cyclist and other endurance athletes, whose sport requires repetitive knee flexion. The majority of cases resolve with conservative treatment. Care in the acute phase focuses on activity limitation, NSAID's, corticosteroids. Analgesia and pain management are two very important aspects in the therapeutic process.

The research project is focused on the dermoelectroporation (pulse current iontophoresis), as a new method for transdermal drug delivery. It was evaluated the feasibility administering the cocktail of Diclofenac Sodium and Dexamethasone Sodium Phosphate by dermoelectroporation (pulse current iontophoresis), for treatment of ITBS. Diclofenac Natrium and Dexamethasone Sodium Phosphate was given locally, via dermoelectroporation (pulse current iontophoresis) in the area of pain (area of the damaged lateral femoral condyle), following microdermabrasion. The therapeutic procedures were performed every day, once a day, 3 times. The pain threshold was recorded objective with Pressure Algometer Somedic in $\mathrm{kPa} / \mathrm{cm}^{2}$. The subjective intensity of the painful sensation was assessed with 10 $\mathrm{cm}$ Visual Analogue Scale (VAS), ranging from $0 \mathrm{~cm}$ (no pain) to $10 \mathrm{~cm}$ (worst possible pain). Using this method of application of nonsteroidal and steroidal anti-inflammatory drugs has significant benefits like: quick pain relief, minimally invasive effect for the patient and minimum side effects. Based on these advantages it is thought that dermoelectroporation could be a preferable way of administering antiinflammatory drugs in inflammatory-degenerative conditions related to the musculoskeletal system, such as overuse injuries like tendinopathies.
\end{abstract}

Key words: dermoelectroporation, iliotibial band syndrome, algometry.

\section{INTRODUCTION}

The iliotibial band (ITB) is the distal fascial continuation of the tensor fascia lata, gluteus medius, and gluteal maximus. Iotibial band syndrome is a common knee injury caused by inflammation of the distal portion of the iliotibial band, which results in lateral knee pain. Iliotibial band syndrome (ITBS) is one of the causes of lateral knee pain and has been diagnosed frequently in long distance runners, cyclists, skiers and participants of basketball and football. Patients with ITBS typically complain of a sharp or burning pain in the area roughly $2-3 \mathrm{~cm}$ superior to the lateral joint line

\footnotetext{
*Correspondence to: Stefan Radev' Department of Physiology, Pathophysiology and Pharmacology Medical Faculty, Trakia University, 11 Armejska Str., 6000 Stara Zagora, Bulgaria'e-mail: radev.st@abv.bg
}

$(1,2)$. The pain may radiate proximally or distally, and in less severe cases, the pain may quickly subside upon cessation of activities (1, 2 ). Often the pain will occur as activities proceed. The athletes have difficulty in performing their routine daily activities when using the affected leg (the activities all depending on rapid and prolonged of the knee through flexion and extension), which in itself leads to a worse quality of life and physical invalidation. ITBS is a clinical diagnosis and most often additional diagnostic studies are not necessary. It should be suspected in overuse and nontraumatic cases of knee pain where rest has not been helpful. The common therapeutic approaches, such as providing rest for the affected leg, local (gels and unguents) and systemic administration of nonsteroidal antiinflammatory drugs, steroid injections, 
physiotherapy, laser therapy, ice application, heat therapy, complete immobilization with a splint, etc., do not lead to satisfactory results in all cases (2-4). The research project is focused on the dermoelectroporation, as a new method for transdermal drug delivery (5). It was evaluated the feasibility administering the combination of Diclofenac Sodium (Voltaren, Novartis) and Dexamethasone Sodium Phosphate (Dexamethasone, KRKA) by dermoelectroporation (pulsed current iontophoresis) for treatment of ITBS (6).

\section{MATERIAL AND METHODS}

The procedure was carried out in 33 individuals (13 males and 20 females) aged between 27 and 56, diagnosed with ITBS, and no other accompanying diseases. Persons with ITBS were recruited from posted flyers and physician offices. Prior to participation, all patients provided written informed consent.

ITBS is a clinical diagnosis and most often additional diagnostic studies are not necessary (4).

All the patients presented with persistent pain and limited activity for more than one week, despite the application of various therapeutic approaches.

Prior to the transdermal drug delivery of the anti-inflammatory drugs, by palpation we determined the area of pain around the affected condyles (7-9). At the same time we measured symmetrically and bilaterally in $\mathrm{kPa} / \mathrm{cm}^{2}$ the threshold of pain in the affected and the healthy area by using Pressure Algometer Somedic, Sweden (10). The pain threshold was recorded objective before every dermoelectroporation procedures and four weeks after therapy. The subjective intensity of the painful sensation was assessed with $10 \mathrm{~cm}$ visual analogue scale (VAS), ranging from 0 $\mathrm{cm}$ (no pain) to $10 \mathrm{~cm}$ (worst possible pain) (11).

A mixture of $75 \mathrm{mg} 3 \mathrm{ml}$ Diclofenac and $4 \mathrm{mg}$ $1 \mathrm{ml}$ Dexamethasone was administered on the first day. The same dose of Diclofenac and 2 $\mathrm{mg}, 0,5 \mathrm{ml}$ Dexamethasone was administered on the second and third day by dermoelectroporation.

The transdermal drug administration dermoelectroporation (pulse current iontophoresis), is utilized locally by using Ultrapeel Transderm Ionto System by Mattioli Engineering, in the area of the affected condylus, following microdermobrasion. Through this method it is possible to perform a controlled exfoliation of the stratum corneum, which increases the skin permeability for the drugs applied in the treated area. The therapeutic procedures were performed every day, once a day, 3 times.

Statistical analyses were conducted using SPSS for Windows Version 16 (SPSS, Inc., Chicago, IL, USA), and level of significance was set at $p<0.05$.

\section{RESULTS}

The threshold of pain sensitivity measured in the contralateral healthy knee joint (PAC) was on the average $848,24 \mathrm{kPa} / \mathrm{cm}^{2} \pm 51,21$, which correlates with the data we obtained in our previous studies (10). The threshold of pain sensitivity measured before first drugs administration was PA1 - 289, $41 \pm 48,83$ $\mathrm{kPa} / \mathrm{cm}^{2}$; before second drugs administration PA2 $523,68 \pm 43,54 \mathrm{kPa} / \mathrm{cm}^{2}, \mathrm{P}=0.091$; before third - PA3 $706,47 \pm 59,58 \mathrm{kPa} / \mathrm{cm}^{2}$ and four weeks later PA4w was $771,76 \pm 51,49 \mathrm{kPa} / \mathrm{cm}^{2}$, $\mathrm{P}=0.089$ (Figure 1).

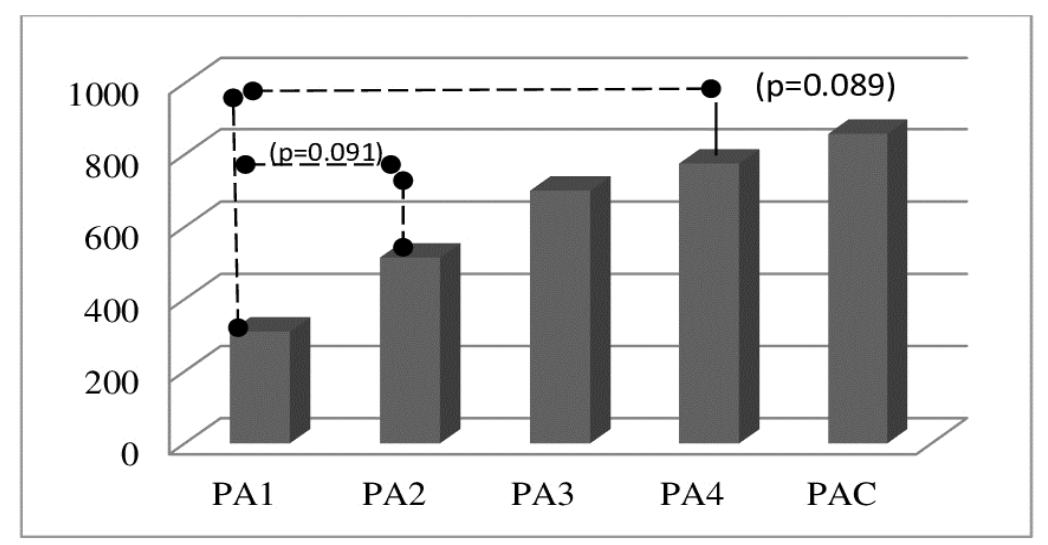

Figure 1. The threshold of pain sensitivity in $\mathrm{kPa} / \mathrm{cm}^{2}$ 
The measured threshold of the pain after the first administration was on the average $61,73 \%$ increase, after the second was on the average $83,28 \%$ increase and after the third it was $90,98 \%$ on the average.
VAS was rated before every therapeutic procedures and four weeks later: VAS1 7,1 см; VAS2 3,9cм; VAS3 1,8 cm; VAS4w - 0,7 cm. $\mathrm{P}=0.028$ (Figure 2).

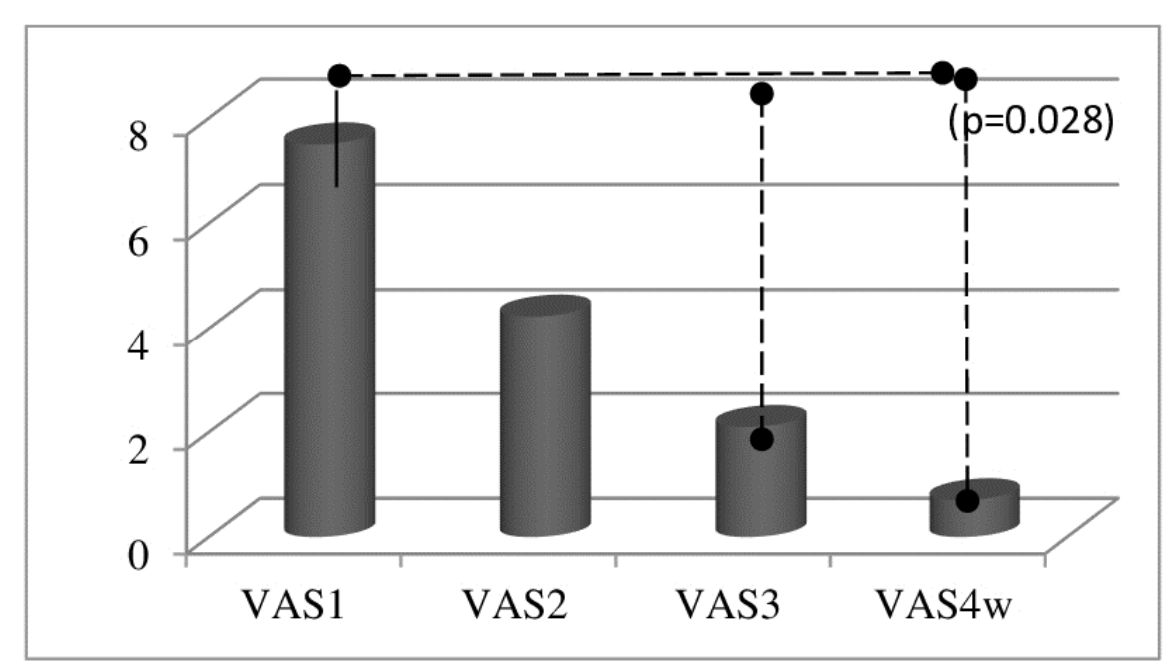

Figure2. Subjective intensity of the painful sensation assessed with Visual Analogue Scale in cm (VAS)

The average VAS pain score decrease to $55,1 \%$ after the first procedures, after second it decrease to $74,49 \%$ and after third (four weeks after therapy), - 90,1\%.

\section{DISCUSSION}

The fast control over the pain in ITBS through this mode of administration of drug combinations is a reliable criterion for the efficacy of the therapeutic application. The non-traumatic approach and the lack of adverse effects are amongst its other advantages. Pain and discomfort are alleviated after the first manipulation, whereas after the second and third the threshold for pain increases up to the values approximating these in a healthy joint. The normal condition of the affected area and the knee is re-established. The long-term perspective is similar to that of the other therapeutic approaches - there always exits a risk of a relapse, especially when intense activity is undertaken immediately after the course of treatment.

\section{CONCLUSION}

The skin is an attractive area for drug delivery due to its prevalence and easy of access. The efficient delivery of drugs into and across the skin has been a goal of researchers for decades. Transdermal drug delivery offers several advantages, including an absence of gastric irritation, painlessness, non-invasiveness, better patient compliance and avoidance of the first - pass metabolism (12). The existent positive therapeutic results in cases of joint and muscle pain, as well as in other regions of the body (5), expand the application of the Ultrapeel ${ }^{\circledR}$ Transderm ${ }^{\circledR}$ Ionto System. It is of clinical-pharmacological interest to find out the lowest effective doses for drug administration in this manner. Such an approach would optimize the use of antirheumatic agents and could also be a promising perspective for the risk groups of patients, in whom the anti-inflammatory drugs of the steroidal and nonsteroidal type are counter indication.

\section{Conflict of interest statement}

There are no conflicts of interest to disclose.

\section{REFERENCES}

1. Pegrum J., Self A., Hall N. Iliotibial band syndrome. BMJ. Mar 2019 21; 364: 1980, doi: 10.1136/bmj. 1980 PMID: 30898786, 2019.

2. Strauss E.J., Kim S., Calcei J. G., Park D. Iliotibial band syndrome: evaluatiom and management. J Am Acad Orthop Surg, 19, (12). 728-736, 2011.

3. M. Fredericson and A. Weir. Practical management of iliotibial band friction syndrome in runners. Clinical Journal of Sport Medicine, vol. 16, 3, 261-268, 2006.

4. Corey Beals and David Flanegan. A review of treatments for Iliotibial band syndrome 
in the athletic population. Journal of Sports, ID 367169,https://doi.org/10.1155/2013/36 7169, Volume 2013.

5. Buselli P., Spaggiari PG. Post traumatic suffering and tendinopathies of sportsmen: a new method for the local pharmacological administration. XIV International Congress on Sports Rehabilitation and Traumatology, 2005.

6. P. Gunter and M. P. Schwellnus. Local corticosteroid injection in iliotibial band friction syndrome in runners: a randomized controlled trial. British Journal of Sports Medicine, vol. 38,(3), 269-272, 2004.

7. Fisher A. A. Pressure algometry over normal muscles. Standard values, validity and reproducibility of pressure threshold. Pain, vol. 30, 15-126,1987.

8. Fisher A. A. Pressure algometry for quantification of diagnosis and treatment outcome. An update. Journal of musculoskeletal pain, vol. 6, (1), 5-32,1998.

9. Ylinen J. Pressure algometry. Aust $J$ Physiother, 53, 207, 2007.

10.St. Radev, Zh. Tsokeva, K. Sokolova, R. Radev. The Algometry by pressure as a method to optimize painrelief therapy. Trakia Journal of Sciences, vol.6, No 2, 146-148, 2008.

11.Jensen M.P., Chen C., Brugger A.M. Interpretation of visual analog scale rating and change scores: a reanalysis of two clinical trials of postoperative pain. J Pain, 4, (7), 407-414,2003.

12.Guy R. H. Intreview with Richard H. Guy. Therapeutic Delivery, 5,(2), $123-128$, 2014. 